\title{
Dosimetry and toxicity outcomes in postoperative high-dose-rate intracavitary brachytherapy for endometrial carcinoma
}

\author{
Eric D. Donnelly, MD', Sunpreet Rakhra, MD', Irene Helenowski, PhD², Mahesh Gopalkrishnan, PhD', John Lurain, MD³, \\ Julian Schink, MD³, Diljeet Singh, MD, Dr Ph', Jonathan Strauss, MD, MBA', William Small Jr., MD', FACRO, FACR, FASTRO \\ IDepartment of Radiation Oncology, 2Department of Preventive Medicine, 3Department of Obstetrics and Gynecology, Robert H. Lurie \\ Comprehensive Cancer Center, Northwestern University Feinberg School of Medicine, Chicago, IL, USA
}

\begin{abstract}
Purpose: The optimal dosimetric parameters and planning techniques for high-dose-rate vaginal brachytherapy (HDRVB) are unclear. Our aim was to evaluate the utility of bladder and rectal dosimetry for patients receiving HDR-VB for postoperative treatment of endometrial carcinoma.

Material and methods: Patients with endometrial cancer who underwent postoperative HDR-VB from January 1 , 2004 through December 31, 2010 were included. All patients underwent primary surgery consisting of total hysterectomy and bilateral salpingo-oophrectomy (TH-BSO) with or without lymph node dissection and were treated with HDRVB without pelvic external beam radiotherapy (EBRT) or chemotherapy. Demographic, pathologic, dosimetric and clinical data were collected.

Results: One hundred patients were identified with the majority of patients receiving HDR-VB in $700 \mathrm{cGy} \times 3$ fractions $(45 \%)$ or 550 cGy $\times 4$ fractions (53\%). No plan was altered based on bladder dosimetry at the time of planning. The rate of acute urinary reactions (<90 days from beginning of RT) grades 1 and 2 were $14 \%$ and $2 \%$, respectively. The rate of late urinary reactions (> 90 days after RT) grades 1 and 2 were $7 \%$ and $3 \%$, respectively. Dose to the bladder point did not correlate with urinary toxicity. No rectal toxicity was reported by patients receiving HDR-VB.

Conclusions: In the setting of HDR-VB without EBRT, the measured dose to the bladder point does not predict urinary toxicity and is very unlikely to indicate the need to change the treatment plan. The treatment of endometrial carcinoma utilizing HDR-VB alone is associated with very low rates of high-grade acute or late bladder toxicity.
\end{abstract}

Key words: endometrial cancer, high-dose-rate, brachytherapy.

\section{Purpose}

In 2011, an estimated 46470 new cases of endometrial cancer were diagnosed and 8120 deaths were attributed to endometrial cancer in the United States [1]. Fortunately, approximately $75 \%$ of women present with stage I disease confined to the uterus [2]. The prognosis in early stage disease is dependent primarily on patient and pathologic risk factors. The mainstay of treatment for early stage endometrial carcinoma is total hysterectomy and bilateral salpingo-oophorectomy (TH-BSO) with or without lymph node dissection followed by selection of patients for postoperative radiotherapy (RT) and/or chemotherapy depending upon risk factors for recurrence. These risk factors include: age, depth of invasion, lymphovascular space invasion, grade, histology and lymph node involvement $[3,4]$. Two large randomized trials demonstrated external beam radiation therapy (EBRT) significantly decreased the rates of both pelvic and vaginal recurrences $[4,5]$. In both trials, the reduction in locoregional recurrences was primarily due to a reduction in the recurrence rate at the vaginal cuff, which accounted for approximately $75 \%$ of recurrences. Based on this recurrence pattern and the side effect profile associated with pelvic EBRT in early stage endometrial cancer, vaginal brachytherapy alone has been evaluated. PORTEC-2 enrolled patients with intermediate-risk endometrial cancer comparing vaginal brachytherapy with pelvic EBRT. Between the two groups there was no significant difference in the rates of recurrence for vaginal brachytherapy and EBRT within the vagina (1.8\% and $1.6 \%)$ or the pelvis $(5.1 \%$ and $2.1 \%$ ) [6]. Vaginal brachytherapy has thus become an increasingly utilized option for postoperative early stage endometrial cancer patients with selected risk factors [7]. 
Vaginal brachytherapy can be delivered utilizing lowdose-rate (LDR) or high-dose-rate (HDR) techniques. HDR vaginal brachytherapy (HDR-VB) offers several logistical advantages over LDR, including the ability to be performed on an outpatient basis, shorter treatment times and less exposure to ancillary staff. The vaginal recurrence rates following HDR therapy have been shown in multiple studies to be less than 3\% [8]. Similarly, the toxicity rate associated with HDR-VB remains low with very limited grade 3 or 4 toxicity [8].

Treatment with HDR-VB has become the most common approach for vaginal brachytherapy [7]. Guidelines are available regarding patient selection, applicator selection, dosimetry, quality assurance, techniques and dose specifications [8]. The optimal dosimetric parameters and planning techniques are unclear. The purpose of this study is to determine the utility of bladder dosimetry for patients receiving HDR-VB for treatment of locally confined early stage endometrial carcinoma.

\section{Material and methods \\ Patients}

Patients were identified using an electronic medical record. Patients with endometrial cancer undergoing postoperative HDR-VB from January 1, 2004 through December 31, 2010 were included. All patients underwent primary surgery consisting of TH-BSO with or without lymph node dissection and were treated at Northwestern Memorial Hospital (Chicago, IL) with HDR-VB without pelvic EBRT or chemotherapy.

\section{Planning technique}

Prior to the first HDR-VB procedure, dosimetric and localization planning was undertaken. Patients underwent a pelvic examination for assessment of the vaginal cuff integrity and to evaluate for signs of residual or recurrent disease. An integrated brachytherapy unit consisting of a dedicated imaging system and brachytherapy patient table was utilized. A Foley catheter was placed in the bladder and a rectal catheter was positioned. Contrast was deposited into the balloon of the Foley catheter and the rectum to enhance visualization of the bladder, rectum and sigmoid column. A vaginal cuff marker or vaginal contrast was placed if necessary for verification of cylinder position. The largest cylinder that could be comfortably accommodated was chosen. The cylinder was positioned in the vaginal canal and orthogonal X-rays were taken to verify and record proper alignment and for future treatment reproducibility. Films were uploaded into the treatment planning software (Nucletron Plato Brachytherapy version $\left.14.2 \cdot 5^{\circledR}\right)$. In instances where the rectal dose approached the normal tissue constraint, a CT was used for treatment planning. Using the contrast for guidance, points were created corresponding to the posterior most aspect of the Foley balloon, the inferior most aspect of the sigmoid colon and the anterior aspect of the rectum. Several rectal points evenly spaced approximately $1 \mathrm{~cm}$ apart were created to cover the span of the rectum abutting the cylinder. Doses to the prescription depth, vaginal surface, rectal, sigmoid colon and bladder points were calculated. The simulation was performed only at the start of the first procedure, as previous studies have not supported repeating dosimetric calculation after the first fraction [9]. The same treatment plan was used thereafter with appropriate correction for source decay.

\section{Treatment technique}

Prior to each additional treatment the vaginal cylinder was repositioned in the same position as the planning session and verified by orthogonal X-rays. RT was delivered using HDR intracavitary brachytherapy. The prescription isodose for treatment was $0.5 \mathrm{~cm}$ from the vaginal mucosa. The prescription fractionation varied by the clinical situation and attending physician preference.

\section{Data collection}

Demographic, pathologic, dosimetric and clinical data were collected. Stage was standardized using FIGO 2009 staging system. Toxicity was scored using the Common Terminology Criteria for Adverse Events (CTCAE) version 4.0. Patients' urinary symptoms were reviewed for each procedure and at each follow-up visit. Acute toxicities were determined by grading severity for the first 90 days and late toxicities beginning 90 days after treatment. From the CTCAE version 4.0 scale, patients received one score ( 1 to 5 ) according to the level of toxicities. The most severe toxicity was recorded for both the acute and late effect. Patients were followed clinically every 3 months for 2 to 3 years, every 6 months through year 5 and annually thereafter.

\section{Results}

One hundred patients meeting entry criteria within the predetermined time frame were identified. The patient and tumor characteristics are outlined in Table 1 . The mean age of the patient population was 62 years (range 25 to 88 years). The majority of the patients included in this study were pathologic stage I (75\%), with stage II and III patients accounting for $18 \%$ and $7 \%$ of the study population, respectively. A single patient with stage IIIC who had refused pelvic EBRT and received HDR-VB alone was included. Of the patients with stage I disease, $87 \%$ were stage IA and $13 \%$ were stage IB. Forty-one percent of patients had grade 1 disease, $29 \%$ of patients had grade 2 disease and $28 \%$ of patients had grade 3 tumors. Most patients had a histological diagnosis of endometriod adenocarcinoma (76\%). Papillary serous histology was identified in $4 \%$, clear cell in 3\%, mixed mullerian histology (carcinosarcoma) in 3\% and adenosquamous histology in $2 \%$ of patients. Mixed histology was encountered in the remaining $12 \%$ of patients.

Treatment details of the vaginal cuff HDR brachytherapy are detailed in Table 2 . Typically, patients were treated with one of two fractionation regimens: 7 Gy $\times 3$ fractions $(45 \%)$ or $5.5 \mathrm{~Gy} \times 4$ fractions $(53 \%)$. A single patient received a reduced total dose of $19.8 \mathrm{~Gy}$ in 3 fractions of 5.5 Gy and a single 3.3 Gy fraction due to an unacceptably high rectal dose. The dose was prescribed to a depth of $0.5 \mathrm{~cm}$ from the vaginal mucosa in all patients. The upper 
Table 1. Patient characteristics $(n=100)$

\begin{tabular}{|c|c|}
\hline Clinical data & $\%$ \\
\hline Mean age (years) & 62.2 (range, 25-88) \\
\hline \multicolumn{2}{|l|}{ Stage: } \\
\hline IA & 65 \\
\hline IB & 10 \\
\hline$\|$ & 18 \\
\hline III & 7 \\
\hline \multicolumn{2}{|l|}{ Grade: } \\
\hline 1 & 43 \\
\hline 2 & 29 \\
\hline 3 & 28 \\
\hline \multicolumn{2}{|l|}{ Histology: } \\
\hline Endometriod adenocarcinoma & 76 \\
\hline Papillary serous & 4 \\
\hline Mixed mullerian (carcinosarcoma) & 3 \\
\hline Clear cell & 3 \\
\hline Adenosquamous & 2 \\
\hline Other & 12 \\
\hline
\end{tabular}

$3 \mathrm{~cm}$ was treated in the majority of patients, with the upper $5 \mathrm{~cm}$ being the target in patients felt to be at greater risk (e.g., papillary serous histology). The mean rectal and bladder point doses were 3.88 Gy and 4.85 Gy, respectively. Equivalent doses were calculated to express the results in terms of equivalent doses given at 2 Gy per day as described in previous studies [10]. Mean total biological equivalent doses for acute and late effects of the bladder were 21.57 Gy ( 2.93 to 47.94 ) and 28.5 Gy (2.53 to 71.76 ). Seven plans were altered due to rectal doses considered to be unacceptably high. Alterations included reducing the dose per fraction, varying source dwell locations/time or repositioning the vaginal cylinder. Four plans (4\%) were altered after the first fraction was delivered to decrease the rectal dose for the re-
Table 2. HDR brachytherapy details

\begin{tabular}{|c|c|}
\hline Details & $\%$ \\
\hline \multicolumn{2}{|l|}{ Total dose (Gy): } \\
\hline 22 & 53 \\
\hline 21 & 45 \\
\hline 19.8 & 1 \\
\hline \multicolumn{2}{|l|}{ Dose/fraction (Gy): } \\
\hline 7.0 & 45 \\
\hline 5.5 & 53 \\
\hline Average treatment time (sec) & 1417.17 \\
\hline \multicolumn{2}{|l|}{ Size of cylinder: } \\
\hline $2.0 \mathrm{~cm}$ & 6 \\
\hline $2.5 \mathrm{~cm}$ & 44 \\
\hline $3.0 \mathrm{~cm}$ & 50 \\
\hline Mean rectal dose per treatment (Gy) & 3.88 \\
\hline Mean bladder dose per treatment (cGy) & 4.854 \\
\hline $\begin{array}{l}\text { Bladder mean treatment equivalent dose } \\
\text { for acute effects (Gy) }\end{array}$ & 21.57 \\
\hline $\begin{array}{l}\text { Bladder mean treatment equivalent dose } \\
\text { for late effects (Gy) }\end{array}$ & 28.50 \\
\hline Surface mean treatment dose for acute effects (Gy) & 54.92 \\
\hline Surface mean treatment dose for late effects (Gy) & 86.60 \\
\hline $\begin{array}{l}\text { Prescription point }(0.5 \mathrm{~mm}) \text { mean treatment dose } \\
\text { for acute effects (Gy) }\end{array}$ & 29.75 \\
\hline $\begin{array}{l}\text { Prescription point }(0.5 \mathrm{~mm}) \text { mean treatment } \\
\text { dose for late effects (Gy) }\end{array}$ & 41.58 \\
\hline
\end{tabular}

maining fractions and three plans $(3 \%)$ were altered prior to the delivery of the first fraction. No plan was altered based on bladder dosimetry at the time of planning.

There were no reports of significant bladder toxicity for the group of patients analyzed. The rate of acute urinary reactions ( $<90$ days from beginning of RT) grades 1 and 2 were $14 \%$ and $2 \%$, respectively. The rate of late urinary reactions

Table 3. Comparisons of categorical demographic variables associations with acute toxicity and with late toxicity (all grades)

\begin{tabular}{|c|c|c|c|c|c|}
\hline & cute toxicity & & & Late toxicity & \\
\hline Stage & $N(\%)$ & $p$-value & Stage & $N(\%)$ & $p$-value \\
\hline$I(N=75)$ & $12(16.0 \%)$ & \multirow[t]{3}{*}{0.57} & $I(N=66)$ & $7(10.6 \%)$ & \multirow[t]{3}{*}{0.34} \\
\hline$\|(N=18)$ & 2 (11.1\%) & & $\|(N=16)$ & $1(6.3 \%)$ & \\
\hline III $(N=7)$ & $2(28.6 \%)$ & & III $(N=7)$ & $2(28.6 \%)$ & \\
\hline Grade & & & Grade & & \\
\hline $1(N=41)$ & 7 (17.1\%) & \multirow[t]{3}{*}{0.73} & $1(N=37)$ & $5(13.5 \%)$ & \multirow[t]{3}{*}{0.37} \\
\hline $2(N=29)$ & $3(10.3 \%)$ & & $2(N=26)$ & $1(3.9 \%)$ & \\
\hline $3(N=28)$ & $5(17.9 \%)$ & & $3(N=25)$ & $4(16.0 \%)$ & \\
\hline Number of Tx & & & Number of Tx & & \\
\hline $3(N=45)$ & $5(11.1 \%)$ & \multirow[t]{2}{*}{0.28} & $3(N=44)$ & $4(9.1 \%)$ & \multirow[t]{2}{*}{0.74} \\
\hline $4(N=55)$ & $11(20.0 \%)$ & & $4(N=45)$ & $6(13.3 \%)$ & \\
\hline
\end{tabular}


Table 4. Comparisons in continuous demographic variables associated with acute toxicity vs. no acute toxicity

\begin{tabular}{lcccccc} 
& \multicolumn{2}{c}{ Acute toxicity } & \multicolumn{2}{c}{ No acute toxicity } \\
\cline { 2 - 7 } & $N$ & Median and range & $N$ & Median and range & $p$-value \\
\hline Dose received (Gy) & 16 & $22(21,22)$ & 84 & $21.5(19.8,22)$ & 0.16 \\
\hline Length of treatment (seconds) & 16 & $1298.5(979,2039)$ & 84 & $1338(654,2504)$ & 0.61 \\
\hline Size of cylinder (cm) & 16 & $3.0(2.0,3.0)$ & 84 & $2.5(2.0,3.0)$ & 0.63 \\
\hline Bladder dose (average, Gy) & 13 & $4.47(2.5,8.38)$ & 74 & $4.78(1.71,9)$ & 0.85 \\
\hline Rectal dose (average, Gy) & 13 & $3.3(1.77,6.35)$ & 82 & $4.03(2.17,6.32)$ & 0.19 \\
\hline Rectal dose (max, Gy) & 14 & $5.80(2.81,10.6)$ & 82 & $5.67(3.16,8.9)$ & 0.59 \\
\hline Equivalent dose for tumor effects (Gy) Bladder & 13 & $20.0(2.9,40.1)$ & 76 & $20.6(6.2,47.9)$ & 0.95 \\
\hline Equivalent dose (late effects no DMF) Bladder & 13 & $24.9(2.5,59.5)$ & 75 & $26.7(6.4,71.8)$ & 0.89 \\
\hline Equivalent dose for tumor effects (Gy) Surface & 13 & $54.7(46.2,78.3)$ & 78 & $54.7(30.3,67.5)$ & 0.95 \\
\hline Equivalent dose (late effects no DMF) Surface & 13 & $88.6(68.7,128.3)$ & 78 & $88.6(38.6,113.2)$ & 0.89 \\
\hline Equivalent dose for tumor effects (Gy) 0.5 mm & 14 & $28.9(28.9,30.3)$ & 84 & $28.9(25.1,58.7)$ & 0.20 \\
\hline Equivalent dose (late effects no DMF) 0.5 mm & 14 & $39.0(39.0,43.8)$ & 84 & $39.0(32.8,96.3)$ & 0.20
\end{tabular}

DMF-dose modifying factor

Table 5. Comparisons in continuous demographic variables associated with late toxicity vs. no late toxicity

\begin{tabular}{lcccccc} 
& \multicolumn{2}{c}{ Late toxicity } & \multicolumn{2}{c}{ No late toxicity } \\
\cline { 2 - 7 } & $N$ & Median and range & $N$ & Median and range & $p$-value \\
\hline Dose received (Gy) & 10 & $22(21,22)$ & 79 & $21(19.8,22)$ & 0.41 \\
\hline Length of treatment (seconds) & 10 & $1399(1056,2504)$ & 79 & $1279(654,2277)$ & 0.26 \\
\hline Size of cylinder (cm) & 10 & $2.8(2.5,3.0)$ & 79 & $2.5(2.0,3.0)$ & 0.69 \\
\hline Bladder dose (Gy) & 9 & $5.03(3.05,8)$ & 70 & $4.85(2.04,9)$ & 0.99 \\
\hline Rectal dose (average, Gy) & 8 & $3.21(2.28,5.98)$ & 76 & $3.92(1.77,6.35)$ & 0.58 \\
\hline Rectal dose (max, Gy) & 9 & $5.82(4.42,10.55)$ & 76 & $5.80(2.81,8.9)$ & 0.62 \\
\hline Equivalent dose for tumor effects (Gy) Bladder & 9 & $20.7(2.9,36.6)$ & 72 & $20.9(6.2,47.9)$ & 0.89 \\
\hline Equivalent dose (late effects no DMF) Bladder & 9 & $27.7(2.5,55.0)$ & 71 & $26.8(6.4,71.8)$ & 0.90 \\
\hline Equivalent dose for tumor effects (Gy) Surface & 8 & $54.7(51.0,78.3)$ & 73 & $54.7(30.3,67.5)$ & 0.93 \\
\hline Equivalent dose (late effects no DMF) Surface & 8 & $88.6(38.6,128.3)$ & 73 & $88.6(43.8,113.2)$ & 0.78 \\
\hline Equivalent dose for tumor effects (Gy) 0.5 mm & 9 & $28.9(28.9,30.3)$ & 78 & $29.6(25.1,58.7)$ & 0.76 \\
\hline Equivalent dose (late effects no DMF) 0.5 mm & 9 & $39.0(39.0,43.8)$ & 78 & $41.4(32.8,96.3)$ & 0.74
\end{tabular}

DMF-dose modifying factor

(> 90 days after RT) grades 1 and 2 were $7 \%$ and $3 \%$, respectively. The urinary reactions included most commonly dysuria, as well as urinary frequency and urinary tract infections, possibly stemming from insertion of the Foley catheter. No grade 3 or higher urinary toxicities were observed. There were no reports of significant rectal toxicity in our patient population. There was no association between any of the recorded patient characteristics and the development of either acute or late toxicity (Table 3). Likewise, none of the evaluated dosimetric variables were found to be associated with the development of acute or late toxicity (Tables 4 and 5). After adjusting for patient characteristics, still no dosimetric variables were found to increase the risk of acute or late toxicity (Table 6).

Overall survival rates at 3 and 5 years were $96 \%$ and $89 \%$, respectively (Fig. 1). Of the 100 patients included in this study, there have been a total of 5 failures (5\%). Two developed locoregional recurrences within the pelvis alone ( 1 nodal and 1 isolated pelvic). Two were found to have distant metastatic disease, both in the lungs. One patient presented with synchronous locoregional and distant disease. There were no vaginal cuff recurrences.

\section{Discussion}

In this single institution series, 100 women treated with HDR-VB displayed excellent local control with low rates of acute or late urinary toxicity. Although bladder dosimetry was performed on all 100 patients, it was uniformly within an acceptable range, was not used to change the treatment plan and did not correlate with risk of urinary toxicity. On review only rectal dosimetry altered total treatment dose 
Table 6. Prognostic factors associated with the odds of experiencing an acute or a late toxicity, adjusted for age, grade, treatment length, stage and histologic type

\begin{tabular}{|c|c|c|c|c|c|c|}
\hline \multirow{2}{*}{$\begin{array}{l}\text { Odds ratio and } \\
95 \% \text { confidence interval } \\
\text { associated with } 5 \text { Gy of: }\end{array}$} & \multicolumn{3}{|c|}{ Acute toxicity } & \multicolumn{3}{|c|}{ Late toxicity } \\
\hline & $\bar{N}$ & $\begin{array}{l}\text { Odds ratio and } \\
95 \% \text { confidence } \\
\text { interval }\end{array}$ & $p$-value & $N$ & $\begin{array}{l}\text { Odds ratio and } \\
95 \% \text { confidence } \\
\text { interval }\end{array}$ & $p$-value \\
\hline Rectal dose (average) & 95 & $0.98(0.95,1.01)$ & 0.31 & 84 & $1.00(0.96,1.05)$ & 0.93 \\
\hline Rectal dose (max) & 96 & $1.00(0.98,1.02)$ & 0.83 & 85 & $1.02(0.99,1.05)$ & 0.20 \\
\hline $\begin{array}{l}\text { Equivalent dose for tumor } \\
\text { effects (Gy) Bladder }\end{array}$ & 89 & $0.99(0.70,1.36)$ & 0.95 & 81 & $0.90(0.55,1.39)$ & 0.63 \\
\hline $\begin{array}{l}\text { Equivalent dose (late } \\
\text { effects no DMF) Bladder }\end{array}$ & 88 & $1.00(0.80,1.22)$ & 0.98 & 80 & $0.96(0.70,1.25)$ & 0.76 \\
\hline $\begin{array}{l}\text { Equivalent dose for tumor } \\
\text { effects(Gy) Surface }\end{array}$ & 91 & $1.26(0.72,2.31)$ & 0.43 & 81 & $1.61(0.84,3.61)$ & 0.18 \\
\hline $\begin{array}{l}\text { Equivalent dose (late effects } \\
\text { no DMF) Surface }\end{array}$ & 91 & $1.13(0.85,1.54)$ & 0.40 & 81 & $1.05(0.77,1.46)$ & 0.74 \\
\hline $\begin{array}{l}\text { Equivalent dose for tumor } \\
\text { effects (Gy) } 0.5 \mathrm{~mm}\end{array}$ & 98 & $0.28(0.01,1.84)$ & 0.45 & 87 & $0.97(0.03,2.82)$ & 0.97 \\
\hline $\begin{array}{l}\text { Equivalent dose (late effects } \\
\text { no DMF) } 0.5 \mathrm{~mm}\end{array}$ & 98 & $0.52(0.14,1.28)$ & 0.29 & 87 & $1.02(0.29,1.73)$ & 0.97 \\
\hline
\end{tabular}

or dose per fraction. These findings are similar to those of other published reports showing that HDR-VB yields excellent rates of local control and toxicity. Multiple series have shown vaginal recurrence rates of $1 \%$ to $2 \%$, with most studies citing no grade 3 or 4 toxicities [8,11-14]. Likely due to these low reported rates of toxicity, $20 \%$ of respondents to an ABS survey indicated that they do not routinely monitor bladder and rectal doses for HDR-VB [7]. In short, the ideal method for calculation of normal tissue dose remains unclear.

The bladder, rectum and vaginal mucosa are organs at risk for possible toxicity in the treatment of endometrial cancer with HDR-VB. The bladder point is defined as the most posterior point in the balloon of the Foley catheter on lateral radiographic film and in the center of the film on an anterior-posterior film. Additional methods used in practice to calculate the bladder dose include computed tomography (CT) scans and in vivo measurements [15]. CT simulation prior to HDR-VB allows evaluation of dose distributions to target volumes and normal structures and the creation of a dose-volume histogram. CT simulation also has the ability to confirm that there are no significant air gaps between the applicator and the vaginal mucosa. Despite these differences, the added benefit of CT simulation as compared to localization with orthogonal radiographs remains debatable. In one study, CT simulation did not alter applicator position or treatment decisions for the vast majority of patients [16]. The utilization of orthogonal X-rays was preferred over CT at our institution based on the potential to quickly obtain verification images in the treatment position immediately prior to treatment without moving the patient or cylinder. In addition, the cost effectiveness of CT simulation has yet to be evaluated.

The goal for all methods of dosimetric calculation is to specify target coverage and to create an accurate estimate

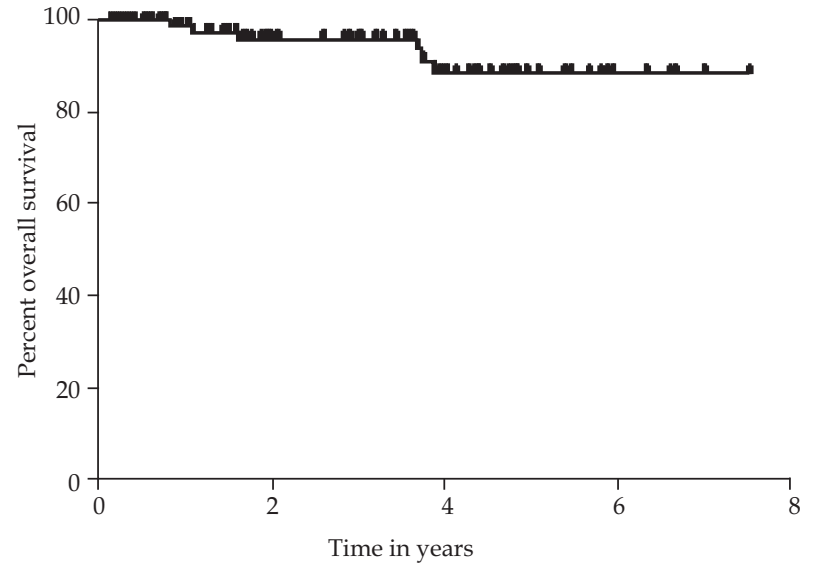

Fig. 1. For all 100 patients analzed, the 3-year survival rate was $96 \%$ (95\% confidence interval, $87.90 \%$, 98.68\%). The 5-year survival rate was $89 \%$ (95\% CI: $75.31 \%, 94.87 \%)$. The mean and standard error of survival time was $3.74 \pm 0.07$ years

of dose received by adjacent organs at risk in order to predict the risk of normal tissue toxicity. In instances where the calculated dose to normal tissue is deemed to be excessive, brachytherapy plans can be adjusted prior to treatment. At our institution, it has been standard practice for patients treated with HDR-VB to have treatment plans created using Foley and rectal catheters and contrast to define the bladder and rectal points. Orthogonal radiographs are then obtained and uploaded to our software for dosimetric planning. The plan created on the first treatment day is subsequently used for the remaining fractions, a strategy referred to as first fraction optimization.

The findings of this study suggest the dose to the bladder point (within a clinically relevant range) does not correlate with the risk of acute or late urinary toxicity from 
HDR-VB. The measurement of the dose to the bladder point failed to indicate the need for a change in the treatment plan of any patients in our series. Finally, the insertion of the Foley catheter may have been the proximate cause for most of the acute urinary reactions seen, namely dysuria and UTIs. For these reasons, consideration should be given to abandoning the use of 2-dimensional bladder dosimetry via Foley catherer during HDR-VB used in the absence of EBRT.

\section{Conclusions}

Established guidelines for HDR-VB include the recommendation to measure the dose to a bladder reference point [8]. The results of the present study suggest that in the setting of HDR-VB without EBRT, the measured dose to the bladder point does not correlate with urinary toxicity and is very unlikely to indicate the need to change the treatment plan. The treatment of endometrial carcinoma utilizing HDR-VB alone is associated with very low rates of highgrade acute or late bladder toxicity. Thus, it may be unnecessary to calculate the bladder reference point during HDR-VB for endometrial carcinoma when administered in the absence of EBRT.

\section{Acknowledgement}

This study was approved by the Institutional Review Board of Northwestern University.

\section{References}

1. Siegel R, Ward E, Brawley O et al. Cancer statistics, 2011. CA Cancer J Clin 2011; 61: 212-236.

2. Creutzberg CL. Endometrial cancer. In: Gunderson L, Tepper JE (eds.). Clinical Radiation Oncology. Elsevier, Philadelphia 2007, pp. 1359-1384.

3. Greven KM, Van Putten WL, Koper PC et al. Which prognostic factors influence the outcome of patients with surgically staged endometrial cancer treated with adjuvant radiation? Int J Radiat Oncol Biol Phys 1997; 39: 413-418.

4. Creutzberg CL, Van Putten WL, Koper PC et al. Surgery and postoperative radiotherapy versus surgery alone for patients with stage I endometrial carcinoma: multicentre randomized trial. PORTEC Study Group. Post Operative Radiation Therapy in Endometrial Carcinoma. Lancet 2000; 355: 1404-1411.

5. Keys HM, Roberts JA, Brunetto VL et al. A phase III trial of surgery with or without adjunctive external pelvic radiation therapy in intermediate risk endometrial adenocarcinoma: a Gynecologic Oncology Group study. Gynecol Oncol 2004; 92: 744-751.

6. Nout RA, Smit V, Putter H et al. Vaginal brachytherapy versus pelvic external beam radiotherapy for patients with endometrial cancer of high-intermediate risk (PORTEC-2): an open-label, non-inferiority, randomized trial. Lancet 2010; 375 : 816-823.

7. Small W Jr, Erickson B, Kwakwa F. American Brachytherapy Society survey regarding practice patterns of postoperative irradiation for endometrial cancer: current status of vaginal brachytherapy. Int J Radiat Oncol Biol Phys 2005; 63: 1502-1507.

8. Small W Jr, Beriwal S, Demanes J et al. The American Brachytherapy recommendations for adjuvant vaginal cuff brachytherapy after hysterectomy. Brachytherapy 2012; 11: 58-67.

9. Holloway CL, Macklin EA, Cormack et al. Should the organs at risk be contoured in vaginal cuff brachytherapy. Brachytherapy 2012; 10: 313-317.
10. Nag S, Gupta N. A Simple method of obtaining equivalent doses for use in HDR brachytherapy. Int J Radiat Oncol Biol Phys 2000; 46: 507-513.

11. Anderson JM, Stea B, Hallum AV et al. High-dose-rate postoperative vaginal cuff irradiation alone for stage IB and IC endometrial cancer. Int J Radiat Oncol Biol Phys 2000; 46: 417-425.

12. Alektiar KM, Venkatraman E, Chi DS et al. Intravaginal brachytherapy alone for intermediate risk endometrial cancer. Int J Radiat Oncol Biol Phys 2005; 62: 111-117.

13. MacLeod C, Fowler A, Duval P et al. High-dose-rate brachytherapy alone post-hysterectomy for endometrial cancer. Int J Radiat Oncol Biol Phys 1998; 42: 1033-1039.

14. Barney BM, MacDonald $\mathrm{KO}$, Lee $\mathrm{CM}$ et al. An analysis of simulation for adjuvant intracavitary high-dose-rate brachytherapy in early-stage endometrial cancer. Brachytherapy 2007; 6: 201-206.

15. Kapp KS, Stuecklschweiger GF, Kapp DS et al. Dosimetry of intracavitary placements for uterine and cervical carcinoma: results of orthogonal film, TLD, and CT-assisted techniques. Radiother Oncol 1992; 24: 137-146.

16. Viswanathan AN, Kiristis C, Erickson BE. Gynecologic radiation therapy novel approaches to image-guidance and management. Springer, New York 2011, pp. 239-245. 